EPJ Web of Conferences 19, 05007 (2012)

DOI: $10.1051 /$ epjconf/20121905007

(C) Owned by the authors, published by EDP Sciences, 2012

\title{
Chemical analysis of ancient relicts in the Milky Way disk
}

\author{
E. Stonkutè ${ }^{1, a}$, R. Ženoviené ${ }^{1}$, G. Tautvaišiené ${ }^{1}$ and B. Nordström ${ }^{2}$ \\ 1 Institute of Theoretical Physics and Astronomy, Vilnius University, Gostauto 12, \\ 01108 Vilnius, Lithuania \\ 2 Niels Bohr Institute, Copenhagen University, Juliane Maries Vej 30, 2100 Copenhagen, \\ Denmark
}

\begin{abstract}
We present detailed analysis of two groups of F- and G- type stars originally found to have similarities in their orbital parameters. The distinct kinematic properties suggest that they might originate from ancient accretion events in the Milky Way. From high resolution spectra taken with the spectrograph FIES at the Nordic Optical Telescope, La Palma, we determined abundances of oxygen, alpha- and $r$-process elements. Our results indicate that the sample of investigated stars is chemically homogeneous and the abundances of oxygen, alpha and $r$-process elements are overabundant in comparison with Galactic disk dwarfs. This provides the additional evidence that those stellar groups had the common formation and possible origin from disrupted satellites.
\end{abstract}

\section{KINEMATIC SUBSTRUCTURES IN THE SOLAR NEIGHBORHOOD}

Observations and theoretical simulations have made much progress and provided us with tools to search for past accretion events in the Milky Way and beyond. The current events are the Sagittarius and Canis Major dwarf spheroidal galaxies, merging into the Galaxy. Traces of such mergers are visible in stellar trails revealed in the Galactic halo by large surveys (e.g. [9]).

Can we also find such traces of ancient merger events in the solar neighborhood? Such events are much harder to discern among the stars of the disk. Combining kinematic and chemical analyses we could answer this question. Helmi et al. (2006), using Nordström et al. (2004) catalog, from correlations between the apocentre (A), pericentre $(\mathrm{P})$ and $z$-angular momentum $\left(L_{z}\right)$ - the so called APL space of orbital parameters - identified three new coherent groups of stars and suggested that those might correspond to remains of disrupted satellites. Stars in those groups cluster around regions of roughly constant eccentricity, they have the distinct distribution of kinematics, metallicity $[\mathrm{Fe} / \mathrm{H}]$ and age, providing evidences of their extragalactic origin.

In our high resolution spectroscopic study we aim to investigate the Milky Way disc. F - and G type dwarf stars are very useful in studing the ancient history of our Galaxy since they are long-lived, and their atmospheres reflect their initial chemical composition. In this study, we measured abundances of oxygen, $\alpha$ - and $r$-process elements and here we discuss our results. The method of detailed chemical analysis, that we used in this study, can be found in our previous paper [8].

\section{DETAILED CHEMICAL COMPOSITION}

From the detailed chemical composition we see that the samples of stars in the investigated groups are chemically homogeneous (Fig. 1). The averaged value of $[\mathrm{Fe} / \mathrm{H}]$ for one of the groups is -0.40

a e-mail: edita.stonkute@tfai.vu.lt

This is an Open Access article distributed under the terms of the Creative Commons Attribution-Noncommercial License 3.0, which permits unrestricted use, distribution, and reproduction in any noncommercial medium, provided the original work is properly cited. 


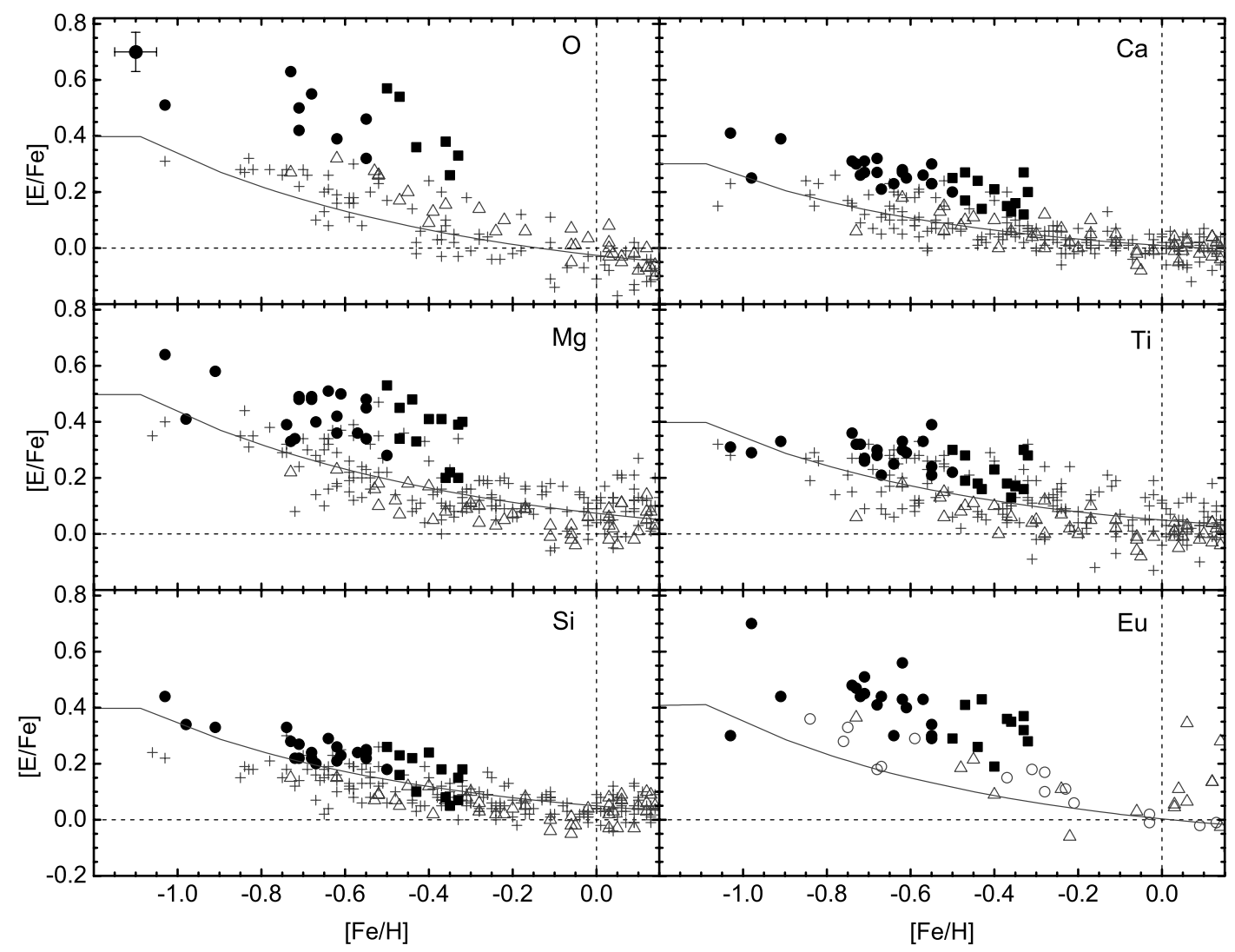

Figure 1. Comparison of chemical element abundance ratios in the investigated stellar groups (black points \& squares), suspected to belong to disrupted satellite(s), and the data for the Milky Way disk dwarfs taken from Edvardsson et al. (1993, plus signs), Bensby et al. (2005, open triangles) and del Peloso et al. (2005, open circles). The Galactic chemical evolution models by Pagel \& Tautvaišienè $(1995,1997)$ are shown by solid lines.

$\pm 0.05 \mathrm{dex}$ and for another is $-0.69 \pm 0.05$ dex. Abundances of oxygen, $\alpha$-elements and $r$-process element europium are overabundant in comparison to the Galactic disk dwarfs [1-3]. The two measured groups of stars have distinct ages of 10 and $12 \mathrm{Gyr}$, respectively. The distinct chemical composition together with the kinematic properties and ages of the stars provide evidences of the ancient merging event(s). Further studies are needed.

\section{References}

[1] Bensby T., Feltzing S., Lundström I., Ilyin I., A\&A, 433, (2005) 185-203

[2] Edvardsson B., Andersen J., Gustafsson B. et al., A\&A, 275, (1993) 101-152

[3] del Peloso E. F., da Silva L. et al., A\&A, 440, (2005) 1153-1159

[4] Helmi A., Navarro J., Nordström B. et al., MNRAS, 365, (2006) 1309-1323

[6] Pagel B.E.J., Tautvaišienè G., MNRAS 276, (1995) 505-514

[6] Pagel B.E.J., Tautvaišienė G., MNRAS, 288, (1997) 108-116

[7] Nordström B., Mayor M., Andersen J. et al., A\&A, 418, (2004) 989-1019

[8] Tautvaišienè G., Edvardsson B., Puzeras E., Ilyin I., A\&A, 431, (2005) 933-942

[9] Yanny B., Newberg H.J., Johnson J., ApJ, 700, (2009) 1282-1298 